\title{
Internal Radioulnar Fixation for Treatment of Nonunion of Proximal Radius and Ulna Fractures in a Toy Breed Dog
}

\author{
Luca Vezzoni $^{1}$ Paolo Abrescia ${ }^{1}$ Aldo Vezzoni ${ }^{10}$ \\ ${ }^{1}$ Clinica Veterinaria Vezzonim, Cremona, Italy \\ VCOT Open 2021;4:e24-e31.
}

Address for correspondence Luca Vezzoni, DVM, Dipl ECVS, GP Cert (SAOS), via Delle Vigne 190, Cremona, CR 26100, Italy (e-mail: luca@vezzoni.it).

\begin{abstract}
In this case report, we describe an alternative surgical procedure to treat proximal radius and ulnar nonunion in a toy breed dog. A 14-month-old, Maltese cross-breed dog was referred after previous treatment with external and internal fixation had failed, resulting in a nonunion of a fracture of the proximal radius and ulna with the proximal radius fragment too small and friable to be used for fixation. A craniomedial approach was made to debride the radius nonunion site and a second approach to the lateral aspect of the ulna was made. The fracture was realigned and a titanium locking plate was applied in bridging fashion, fixed to the proximal ulnar fragment with three locking screws in the most proximal plate holes, a fourth screw was inserted in the mid-shaft of the distal ulnar fragment and three locking screws were inserted in the distal most holes of the plate through the distal ulna to engage the distal radial fragment. A recombinant bone morphogenetic protein- 2 graft was inserted into the radius and ulna fracture sites. The dog had a successful clinical and radiographic outcome with bridging of the defect 4 weeks postoperatively and complete callus formation 8 weeks

Keywords

- locking plates

- fracture

- radius ulna postoperatively. Implants have undergone dynamization and then removal. Use of a locking plate as an internal fixator achieving fixation of the proximal ulna and distal radius can be considered an option for the treatment of proximal radioulnar nonunions with a small proximal radial fragment.
\end{abstract}

\section{Introduction}

Fractures of the radius and ulna are common in dogs, particularly in miniature and toy breeds, ${ }^{1-4}$ and many fixation methods have been described. Conservative treatment using rigid bandages in toy breed dogs commonly results in complications including malunion or nonunion in up to $80 \%$ of fractures. ${ }^{5,6}$ External skeletal fixation has been successfully used to manage radius fractures in small breed dogs, but specific postoperative management and frequent follow-up examinations require owner compliance. ${ }^{7-9}$ Bone plating is a popular method for fixation of radius and ulna fractures ${ }^{10-13}$;

received

December 25, 2020

accepted after revision

May 3, 2020
DOI https://doi.org/

10.1055/s-0041-1723831.

ISSN 2625-2325. however, complications can occur in up to $54 \%$ of dogs that weigh less than $6 \mathrm{~kg}^{14,15}$ These complications include delayed union, nonunion, re-fracture after implant removal and osteopenia due to stress protection. ${ }^{16,17}$ Nonunion is an interruption of the fracture healing process, which necessitates surgical intervention to allow normal healing. Nonunion fractures are characterized by formation of fibrous or cartilaginous tissue between fragments. This is a serious complication in small animal orthopaedics, particularly in the treatment of radius and ulna fractures in toy breed dogs. Nonunion occurs in the radius and ulna in $60 \%$ of cases, in the tibia in $25 \%$ and in the femur in $15 \% .{ }^{18-20}$ Several factors contribute to nonunion,

\section{(c) 2021. The Author(s)}

This is an open access article published by Thieme under the terms of the Creative Commons Attribution License, permitting unrestricted use, distribution, and reproduction so long as the original work is properly cited. (https://creativecommons.org/licenses/by/4.0/)

Georg Thieme Verlag KG, Rüdigerstraße 14, 70469 Stuttgart, Germany 
including inappropriate surgical treatment, instability of fracture sites, infection and poor blood supply. Therapeutic planning for septic nonunion fractures must consider several factors, such as local blood supply, mechanical stability and regenerative ability of the involved tissues. The surgical treatment of nonunion fractures remains a therapeutic challenge for orthopaedic surgeons, especially in the presence of infection, bone loss or both. ${ }^{17}$

One of the primary objectives in treating septic nonunion fractures is adequate debridement to reduce bacterial load and to remove necrotic tissue and sequestra. The debrided fracture must be re-stabilized with appropriate internal or external fixation, providing adequate inter-fragmentary compression if bone apposition is possible. ${ }^{18-20}$ Enhancement of fracture healing and bone defect filling are important steps that can be accomplished with a variety of materials including autologous or allogeneic cancellous bone grafts, demineralized bone matrix, artificial bone materials such as $\beta$-tricalcium phosphate and growth factors such as bone morphogenetic proteins (BMPs). ${ }^{21-30}$

Fractures and nonunion of the proximal radius occur infrequently but are particularly challenging because of the anatomic constraints to surgical approach and limited bone stock for implant fixation. ${ }^{4}$ When the proximal fragment of the radius is too small to achieve adequate fixation, treatment options include use of an interlocking nail or a bone plate on the ulna only, or the use of an external skeletal fixator with fixation pins applied to the olecranon and to the distal radius fragment. ${ }^{4,31,32}$ The purpose of this report was to describe the use of a locking plate fixed to the proximal ulna and distal radius for treatment of nonunion of proximal radius and ulna fractures in a toy breed dog.

\section{Case Report}

\section{Clinical History}

A 14-month-old, $4.5 \mathrm{~kg}$, Maltese cross-breed dog was referred because of nonunion of a fracture of the proximal radius and ulna of the left limb. The dog had sustained a mildly comminuted fracture of the left proximal radius and ulna at 12 months of age after being bitten by a dog (-Fig. 1A). The fracture had been initially treated with an external skeletal fixator. (-Fig. 1B). Three weeks later, surgical revision at the same veterinary practice was done to remove the external skeletal fixator and place intramedullary pins in the radius and ulna (-Fig. 1C). Those implants were subsequently removed 1 month later because of implant failure, and the limb was then splinted ( - Fig. 1D).

The dog was admitted to our clinic 2 months after the initial injury with non-weight-bearing lameness of the left forelimb. Mediolateral and craniocaudal radiographic views revealed nonunion of the proximal radius and ulna fractures with periosteal reaction on the distal fragment of the radius and a suspected bone sequestrum. The proximal fragment of the radius was $4 \times 5 \mathrm{~mm}$ (-Fig. 2 A).

Clinical evaluation of the leg after splint removal revealed skin lesions, severe instability of the fracture site and generalized muscle wasting of the limb (-Fig. 2B). The dog was treated with a broad-spectrum antibiotic medication (amoxicillin + clavulanic acid, Synulox: Pfizer, Rome, Italy, $20 \mathrm{mg} / \mathrm{kg}$ orally three times a day [TID]) without performing a culture and sensitivity test, the skin lesions were medicated daily and the limb was protected with a modified RobertJones bandage. Ten days after referral, the skin lesions had completely healed, and radiographic evaluation showed remodelling of the periosteal reaction. The results of a complete blood cell count, serum biochemical profile and urinalysis were unremarkable. Surgical revision was then performed.

\section{Revision Surgery}

The dog was premedicated with fentanyl (Fentanest: Actavis Italy S.p.A, Nerviano, Italy, $4 \mu \mathrm{g} / \mathrm{kg}$, intramuscularly [IM]), morphine (Morfina Cloridrato: Molteni, Italy, $0.15 \mathrm{mg} / \mathrm{kg}$, IM) and acepromazine (Prequilan!, Fatro SpA, Ozzano Emilia [BO], Italy, $0.02 \mathrm{mg} / \mathrm{kg} \mathrm{IM).} \mathrm{Anaesthesia} \mathrm{was} \mathrm{induced} \mathrm{with}$ propofol (2-4 mg/kg intravenously [IV]) and maintained with a mixture of isoflurane and oxygen (IsoFlo: Aesica Queenborough Limited, Kent, United Kingdom) after endotracheal intubation. A constant rate infusion of fentanyl (Fentanest: Actavis Italy S.p.A, Nerviano, Italy, $10 \mathrm{mcg} / \mathrm{kg} / \mathrm{h}$ ) provided analgesia, and cefazolin (Cefazolina Dorom: Teva Pharma Italia, Milano, Italy, $20 \mathrm{mg} / \mathrm{kg}$, IV) was administered 1 hour before surgery and repeated 120 minutes later. The anaesthetized dog was positioned in dorsal recumbency, and the limb was aseptically prepared in a hanging position.

A standard craniomedial approach at the level of the fracture site was used to remove the sequestrum and to debride the fracture fragments (-Fig. $\mathbf{3 A}$ ) ${ }^{33}$ The proximal radius fragment was too small and friable to be used for fixation. The fracture site was swabbed for bacterial culture and susceptibility testing. A second incision extending from the proximal olecranon to the ulnar styloid was then made on the lateral side. After caudal retraction of the flexor carpi ulnaris muscle and cranial retraction of the ulnaris lateralis muscle, the fracture was manually realigned, but no attempt was made to reduce the radial head. An 11-hole titanium advanced locking plate (ALPS 6.5, Kyon, Zurich, Switzerland) was applied in bridging fashion to the proximal ulnar fragment with three $2.4-\mathrm{mm}$ locking screws in the three most proximal plate holes. A fourth screw was inserted in the mid-shaft of the distal ulnar fragment. Three 2.4-mm locking screws were inserted in the distal most holes of the plate through the distal ulna to engage the distal radial fragment (-Fig. 3B). A recombinant bone morphogenetic protein-2 (rhBMP-2) graft was prepared according to the manufacturer's instructions by applying $1.4 \mathrm{~mL}$ of solution to an absorbable collagen sponge (TruScient; Zoetis Inc., Madison, New Jersey, United States), which was left to soak for 15 minutes before application. Two-thirds of the prepared sponge were inserted into the radius and ulna fracture sites (-Fig. 3C). The tissue layers were routinely closed, and a modified Robert Jones bandage was applied to the limb.

Postoperative radiographic views showed appropriate implant positioning and satisfactory alignment of the radius and ulnar fracture segments, with the exception of a mild 

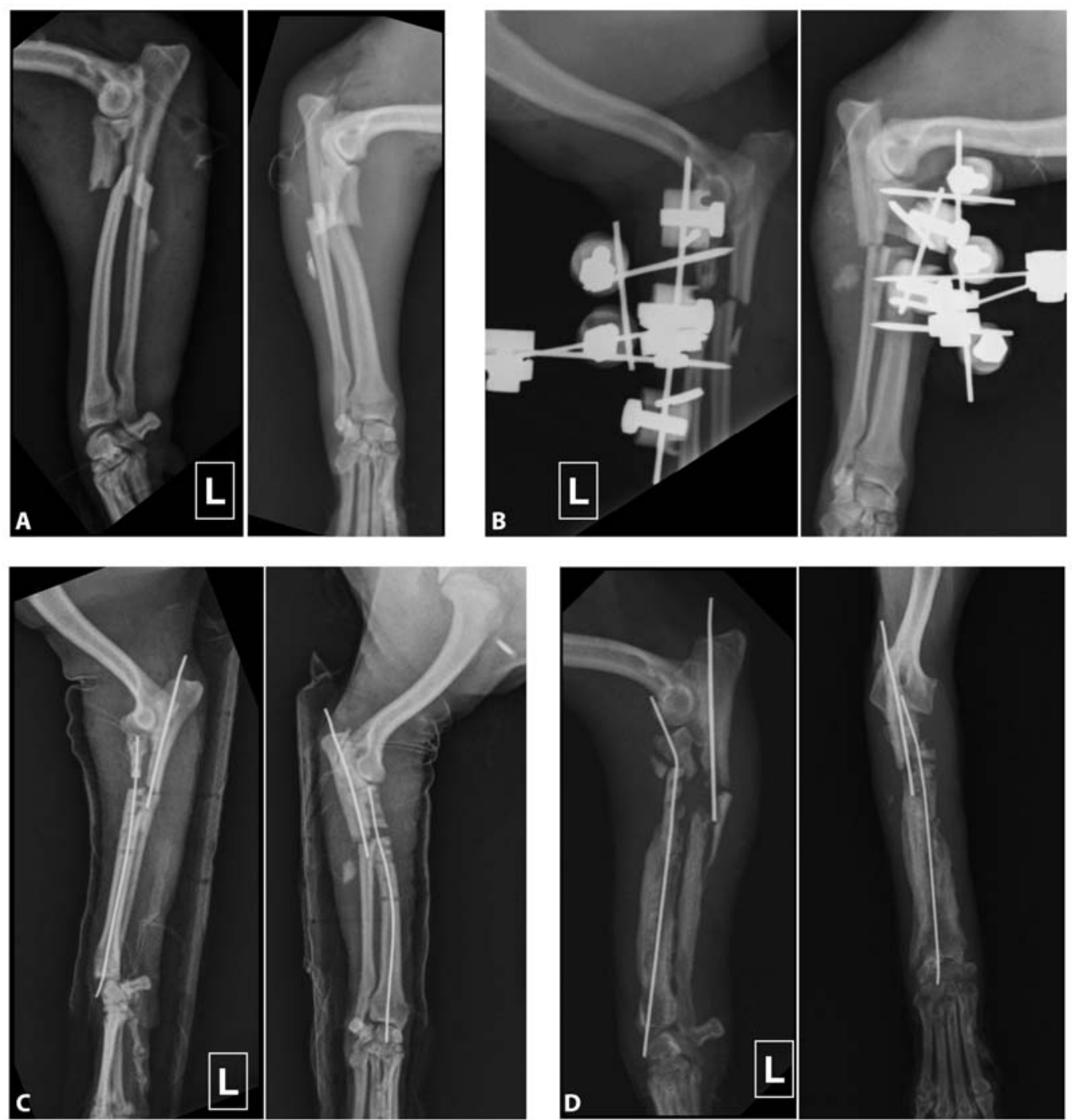

Fig. 1 Radiographs obtained at the primary veterinary care clinic. (A) Preoperative images show a mildly comminuted fracture of the left radius and ulna. (B) Postoperative images after application of an external skeletal fixator. (C) Postoperative images after application of intramedullary pins. (D) Radiographs at the time of failure of intramedullary pinning showing bending of the radial pin and invasion of the elbow and carpal joints.

recurvatum (-Fig. 4A). A 3-mm gap was evident between the proximal and distal fracture fragments. The overall length of the radius from the radial head to the radiocarpal joint was $6.45 \mathrm{~cm}$, compared with $7.5 \mathrm{~cm}$ in the contralateral $\operatorname{limb}$ (-Fig. 4B and C).

The dog was hospitalized for 1 week after surgery. Amoxicillin with clavulanic acid (Synulox: Pfizer, Rome, Italy, $20 \mathrm{mg} / \mathrm{kg}$ orally TID) was administered for 5 days postoperatively and was then discontinued because bacteriological culture results were negative. Meloxicam(Metacam: Boehringer Ingelheim Vetmedica $\mathrm{GmbH}$, Ingelheim, Germany, $0.1 \mathrm{mg} / \mathrm{kg}$ orally) was administered once daily for 1 week and then on alternate days for another 2 weeks. The bandage was removed 2 days after surgery to assess wound healing, which proceeded uneventfully, and then replaced. The dog was discharged 7 days after surgery with instructions to restrict activity to in-house confinement and short leash walks for 4 weeks, after which time the amount of exercise was gradually increased. Wound healing was uneventful and the skin sutures and bandage were removed 12 days postoperatively.
Re-evaluation 4 weeks after surgery revealed persistent moderate weight-bearing lameness of the left forelimb. The range of motion of the elbow was normal, but the carpal range of motion was moderately reduced. Mediolateral and craniocaudal radiographic views (-Fig. 5A) showed progression of bone healing with interfragmentary callus formation that was starting to bridge the fracture gap, but the fracture lines were still visible.

Re-evaluation 8 weeks postoperatively revealed mild intermittent weight-bearing lameness. The same radiographic views showed complete healing of the fracture (-Fig. 4B). The radiopacity of the radial shaft, particularly the distal third, appeared decreased, which was thought to be attributable to stress shielding. For this reason, dynamization of the implant by removal of two screws in the distal radial fragment and another screw in the distal ulnar fragment was done through separate stab incisions (-Fig. $4 \mathbf{C}$ ).

Re-evaluation 20 weeks after surgery revealed mild intermittent weight-bearing lameness with moderate palmigrade stance. Radiographic evaluation showed remodelling of the 


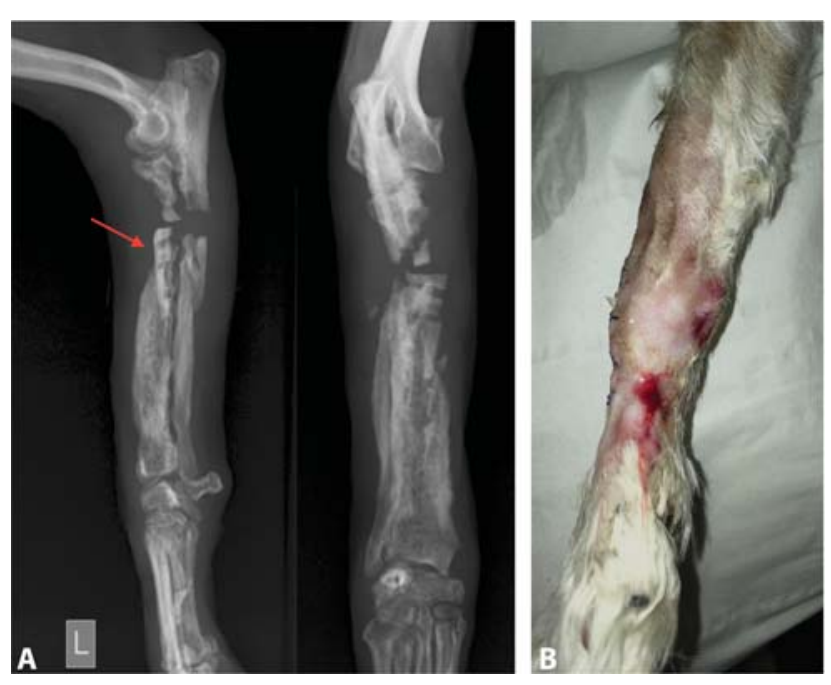

Fig. 2 (A) Radiographic evaluation performed at the time of presentation showing nonunion of radioulnar fractures, the presence of a sequestrum (red arrow) and periosteal reaction on the distal radial fragment. (B) Image taken after removal of the splinted bandage showing skin lesions on the caudal and lateral aspects of the antebrachium.

fracture callus, increased radiopacity of the radius and ulna and signs of degenerative changes at the level of the radiocarpal joint ( - Fig. 4D). The dog underwent implant removal, and postoperative radiographs showed radioulnar synostosis at the level of the proximal radius ( $\mathbf{- F i g . ~} \mathbf{4 E}$ ).

A final re-evaluation was done 36 weeks after surgery. The dog had mild intermittent lameness and a moderate palmi- grade stance. The elbow range of motion was normal and manipulation elicited no pain, but the range of motion of the radiocarpal joint was decreased during flexion and increased during extension. Radiographs showed progression of the degenerative changes at the radiocarpal joint (-Fig. 4F).

\section{Discussion}

A serious complication of fracture treatment is nonunion, which has a higher rate in radioulnar fractures in toy dog breeds because of mechanical and biological factors. ${ }^{34}$ In our case, initial osteosynthesis using external fixation was inadequate and intramedullary pinning in a subsequent revision surgery led to development of a nonviable necrotic nonunion. Guidelines for the treatment of this type of nonunion include removal of necrotic bone fragments, debridement of fracture edges until bleeding from the periosteum and endosteum is observed, grafting with cancellous bone, bone substitutes or growth factors and providing stable external or internal fixation. ${ }^{18-20}$ Regeneration of new bone, particularly large amounts, ensuring the shape of the newly formed bone is adequate and overcoming soft tissue problems associated with nonunion are challenging. Amputation may be the choice in many cases because of financial burden and a guarded prognosis with respect to achieving normal limb function.

Transverse fractures or nonunion of the radial neck can be stabilized with small T-plates when the radial head fragment provides sufficient bone for the placement of two screws. More comminuted radial head and neck fractures, proximal fragments that are too small to achieve stable fixation or
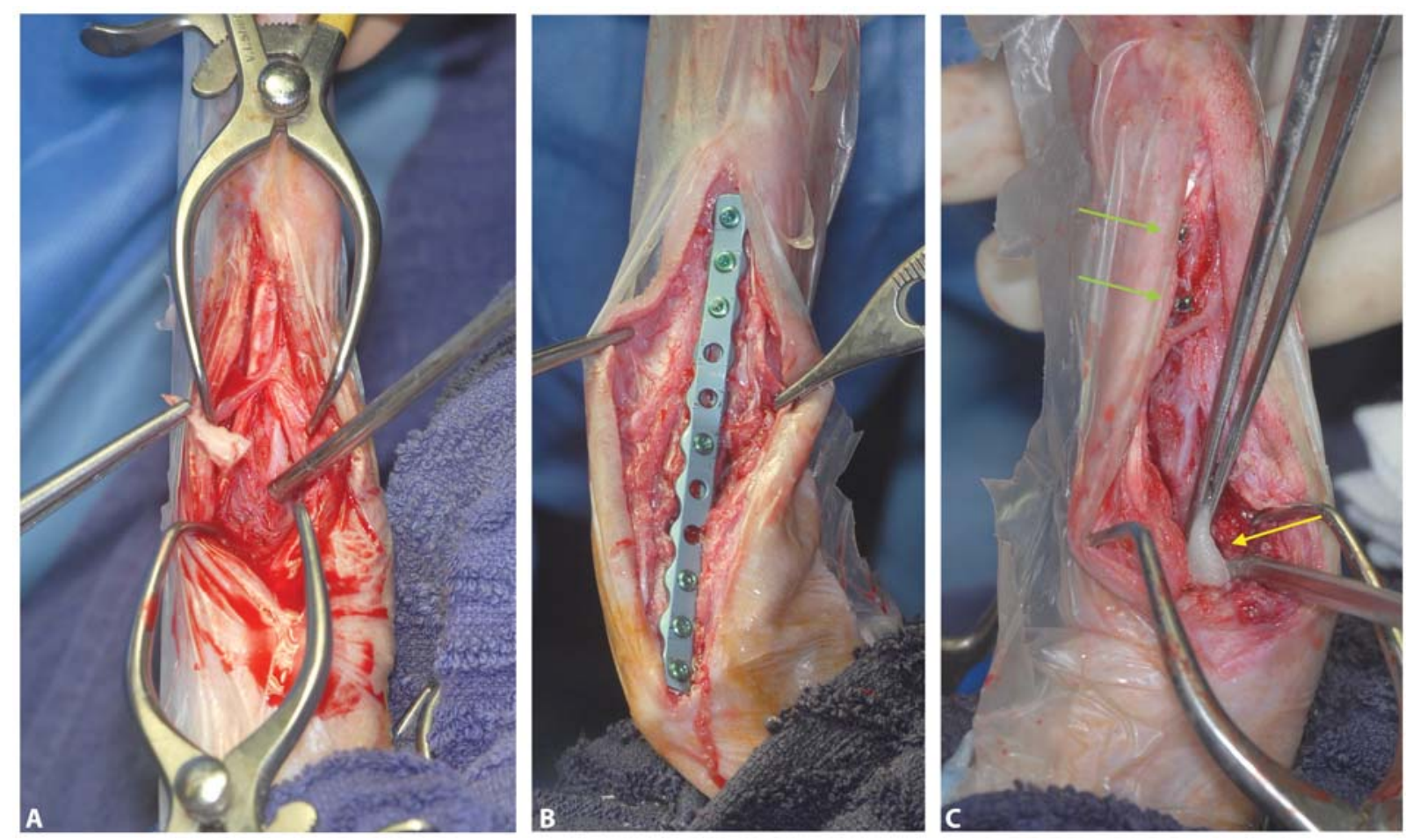

Fig. 3 Intraoperative images. (A) Removal of the sequestrum via a craniomedial approach. (B) Application of the twisted bone plate on the lateral surface of the ulna. (C) Application of the recombinant bone morphogenetic protein-2 graft delivered in an absorbable collagen sponge (yellow arrow). Note the tip of two screws protruding from the medial cortex of the distal radius, confirming proper orientation of the screws through the distal ulna (green arrows). 

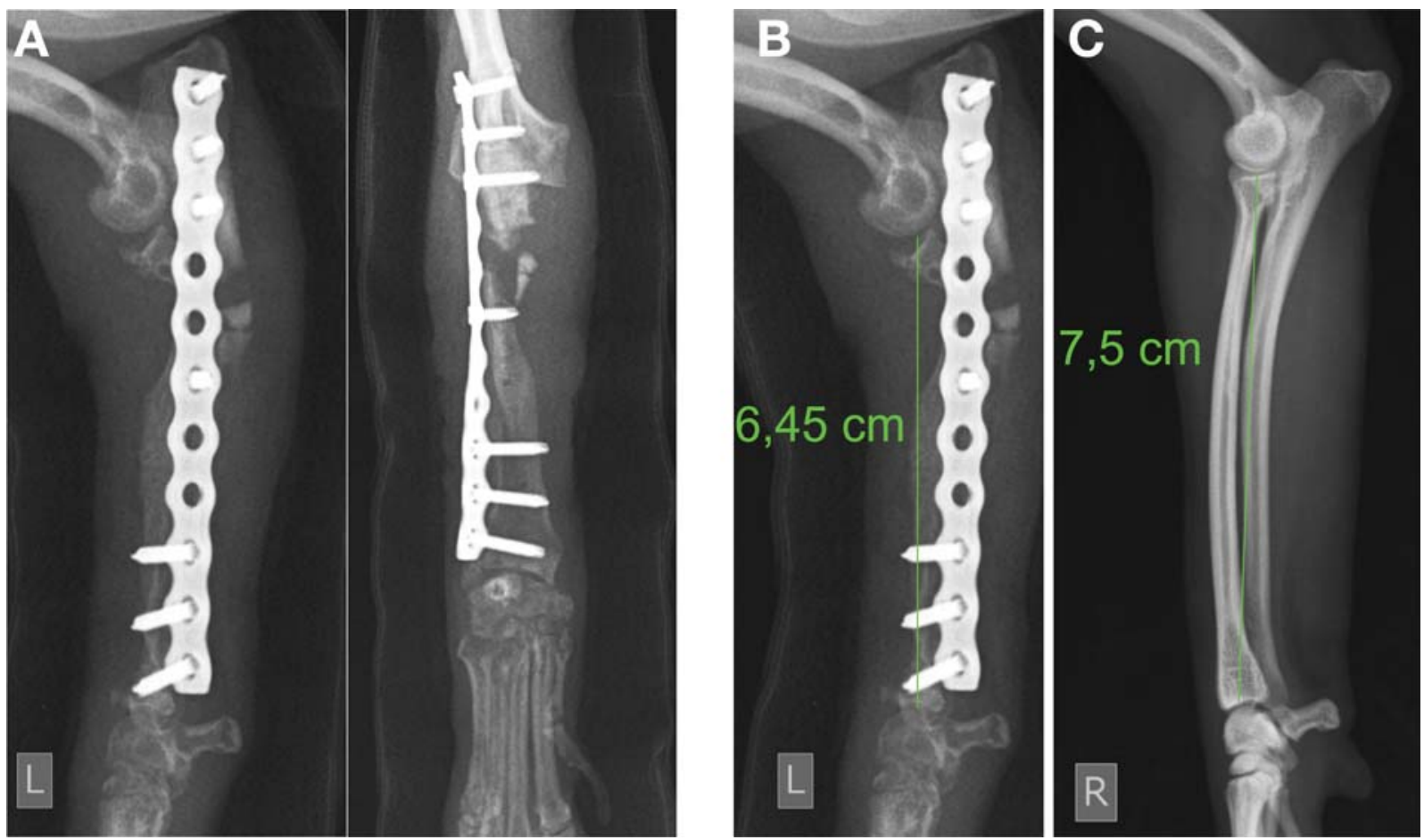

Fig. 4 Immediate postoperative radiographs showing proper implant positioning and alignment (A). Fourteen percent length discrepancy is evident between the operated limb (B) and the contralateral limb (C).

fractures that offer little opportunity for load sharing with internal fixation will likely require additional stabilization. This includes the placement of a circular external skeletal fixator or a hybrid-circular external skeletal fixator with wires that run from a proximal ring to engage the radial head. ${ }^{4}$ In our case, because of the iatrogenic damage resulting from previous surgeries, the radial head did not provide sufficient bone for implant placement and therefore an alternative technique was needed. Ideally the proximal ulna would be fixed to the distal radius allowing the proximal radius to 'float' while still attached to the ulna by soft tissue structures (annular ligament, cranial crura of the collateral ligaments and joint capsule) to maintain its position. This can be achieved by using an external skeletal fixator that engages the proximal olecranon and extends to the radius and ulna distal to the fracture or by simply stabilizing the ulna alone with a bone plate or interlocking nail. ${ }^{4,31,32}$

In toy breed dogs, the shape of the distal ulna precludes stable fixation using a bone plate on the ulna alone, and the small diameter of the medullary canal of the distal ulna does not allow insertion of an interlocking nail. ${ }^{35}$ Therefore, we felt that proximal fixation of the olecranon and distal fixation of the radius was the best treatment option. The use of an external skeletal fixator was not chosen because of reluctance of the owners to provide postoperative home care and the aggressive nature of the patient.

Locking bone plates, also called fixed angle implants, have been developed over the past two decades in an attempt to overcome limitations of conventional plates and screws. Plates with a fixed-angle locking system do not require bone-plate contact to achieve stable fixation, which aids in maintaining desired fragment positioning and eliminates the need for plate contouring. This type of implant aligns with the philosophy of biological osteosynthesis, which highlights functional alignment, relative fracture stability and promotion of an optimal biological environment to promote fracture healing. The biomechanics of locking plates and screws is similar to that of external skeletal fixators and their transfixation pins; locking screws act as transverse supporting components subjected to cantilever bending. The angular stability of the construct converts shear stress created during axial loading or bending to compressive stress at the screwbone interface. ${ }^{36}$

Several locking plate systems are commercially available and currently used at our clinic. The ALPS system was chosen because of its material properties and ease of contouring, which can be accomplished in three planes. The plates are made of grade 4 titanium (Cp Ti, ASTM F- 67, ISO 5832-2) and the screws are made of titanium alloy (Ti-6A1-4V, ASTM F1472, ISO 5832-3). ${ }^{37}$

An experimental study on rabbits showed increased resistance to localized infection when titanium implants were used, and we therefore decided that this material would be of benefit in our patient with necrotic nonunion. ${ }^{38}$ Even when results of culture and sensitivity testing are negative, nonunion attributable to the presence of bacteria is possible and likely underdiagnosed. A study that compared molecular diagnostics and traditional culture methods in 24 human patients with nonunion found bacteria via molecular diagnostics in 30 samples but positive culture results in only eight. ${ }^{39}$

The plate was positioned on the lateral surface of the proximal and distal ulnar fragments and contoured. The lateral surface of the ulna is straight and thus only minor contouring was required to restore alignment in the frontal 

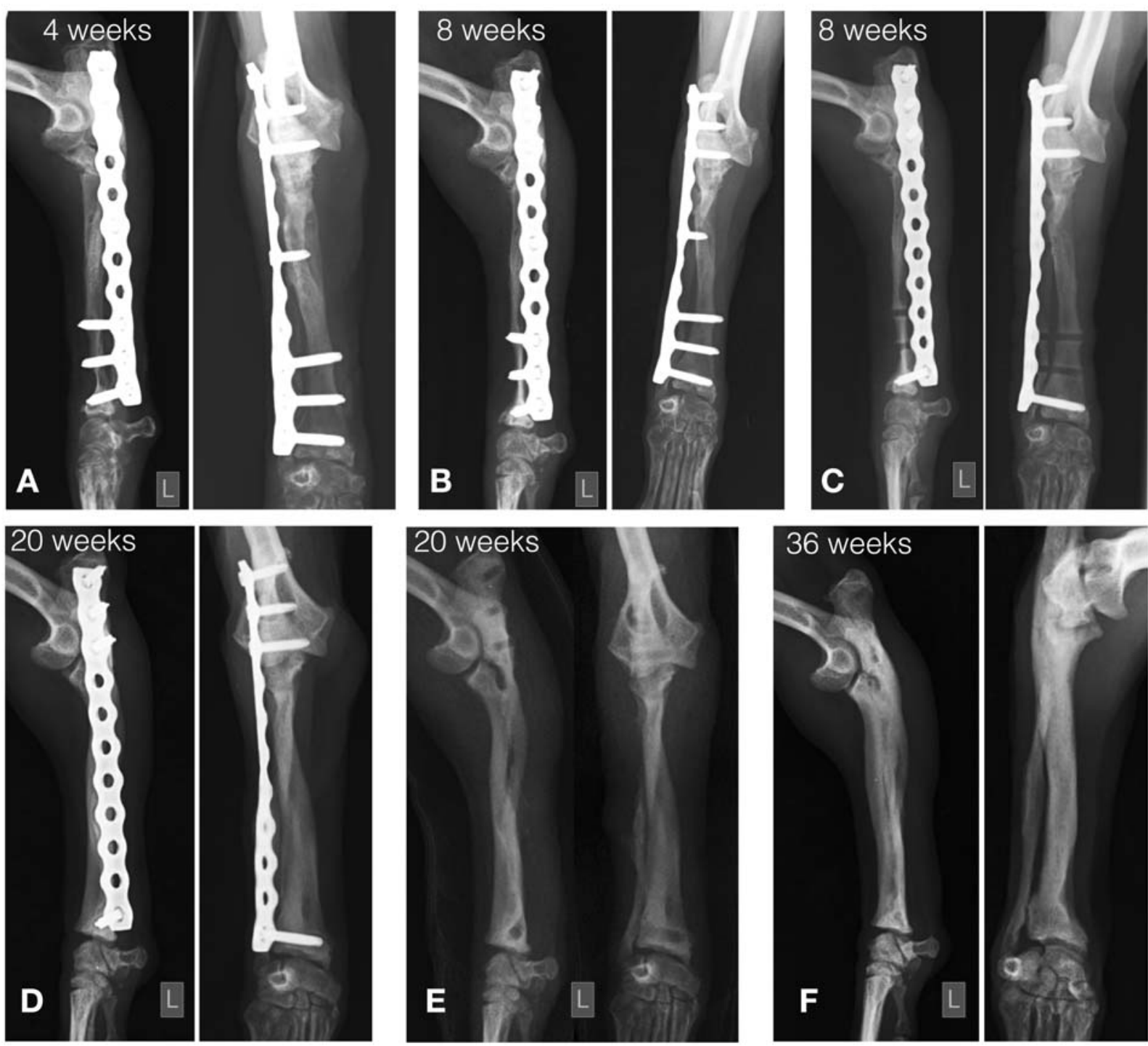

Fig. 5 Radiographs taken at postoperative re-evaluation. (A) Four weeks after surgery, bridging of the fracture gap is evident but fracture lines are still visible. (B) Eight weeks after surgery, complete fracture healing is evident. Decreased bone density at the level of the radial and ulnar diaphysis attributable to stress protection is seen and implant dynamization was undertaken (C). Twenty weeks after surgery, improved bone density is evident (D). Radioulnar synostosis is visible after implant removal (E). Thirty weeks after surgery, fracture callus appears remodelled and degenerative changes are evident at the radiocarpal joint.

plane, while a more pronounced 'on plane' contouring could have been made to avoid the mild iatrogenic recurvatum that resulted from the application of the straight plate. Locking screws need to be inserted perpendicular to the plate, which means the direction of the screw cannot be adjusted. Because the distal ulna is positioned slightly caudal with respect to the distal radius, the plate had to be twisted to direct the screws through the ulna and into the distal radius in a caudolateral to craniomedial direction. ${ }^{35}$ The degree of twisting was determined visually after insertion of the proximal screws. The use of a polyaxial locking plate would have made orientation of the distal screws easier, but this system was not available at our clinic at that time. ${ }^{36,40}$ Another option would have been to contour the plate so that its distal aspect fits the cranial surface of the radius. However, we used the lateral surface of the ulna for plate placement because application was easier and it eliminated the need for more aggressive dissection of tissues and more surgical time. The plate in this configuration acts as a uniplanar unilateral linear external skeletal fixator, which is the least stiff configuration of an external skeletal fixator. The locking plate, however, is relatively closer to the bone than the connecting bar of an external skeletal fixator, providing increased mechanical advantage for the construct. ${ }^{36}$

Different grafting techniques are effective in treatment of nonunion, and we used rhBMP-2 delivered in a collagen sponge. The BMPs initiate a cascade of developmental events, in which pluripotent mesenchymal cells are induced to differentiate into osteoprogenitor cells, osteoblasts and ultimately into osteocytes thus resulting in new bone formation. ${ }^{41-44}$ The BMPs were effective in healing of experimentally-induced osteotomy and for repair of mandibular defects and nonunion in dogs. ${ }^{27-29}$ Successful use of rhBMP-2 depends on the 
quantity and concentration of the product, time of application and incorporation of a delivery vehicle. A single product approved for augmentation of fracture healing in dogs was available in the European Union (TruScient; Zoetis Inc., Madison, New Jersey, United States), which is rhBMP-2 $(0.2 \mathrm{mg} / \mathrm{mL})$ delivered in an absorbable collagen sponge. A paucity of data on the dose of rhBMP-2 used in clinical cases renders comparison of results difficult. When the dose of rhBMP-2 is too high, hypertrophic bone and soft tissue inflammation may occur. ${ }^{45}$ We found that using two-thirds of an absorbable collagen sponge soaked in $1.4 \mathrm{~mL}$ of solution provided rapid filling of the bone defect with formation of abundant bone callus at the 4-week re-evaluation. Immediate postoperative soft tissue swelling adjacent to the graft is a potential complication of rhBMP-2 because of an associated increase in angiogenesis. Leakage of high protein transudate from a large number of new blood vessels is thought to be the source of rhBMP-2-induced postoperative swelling when the product is used at pharmacological doses. Excessive soft tissue swelling is associated with an increased risk of incisional dehiscence. ${ }^{45}$ In our case, there were no signs of soft tissue swelling at the time of bandage change 2 days after surgery and wound healing proceeded uneventfully.

We elected staged removal of the implants because of suspected osteopenia of the diaphyseal bone secondary to stress protection seen on radiographs at the 8-week re-evaluation. Stress-protection osteopenia occurs when bone undergoes cortical atrophy because of subphysiological loading of the bone associated with implants or external devices. ${ }^{46}$ In toy breeds, stress-protection osteopenia is a concern and thus the standard of care at our clinic includes staged removal of implants to avoid this complication. In this case, the positioning of a screw in the proximal aspect of distal ulnar fragment, shortening the working length of the plate, could have contributed in increasing the stiffness of the construct. Dynamization or sequential removal of the screws and plate allows a gradual increase in strain and a controlled increase in load to stimulate remodelling in the affected bone. ${ }^{47,48}$ Dynamization was effective in promoting an increase in bone density and eliminating the radiographic signs of osteopenia in our case, but required two additional operations with all of the associated costs and risks.

Compared with the contralateral limb, severe bone loss resulted in a total length reduction of $14 \%$ in the left radius of our patient. To the authors' knowledge, data on the amount of discrepancy between the length of the forelimbs that is acceptable without causing significant lameness are not available. Dogs can tolerate up to $20 \%$ reduction in femoral length by increasing the standing angle of the stifle and hock. ${ }^{49}$ It can therefore be presumed that a certain amount of discrepancy in forelimb length can be overcome by increasing the standing angle of the shoulder and elbow. The mild intermittent lameness and moderate palmigrade stance seen at the final re-evaluation were likely caused by degenerative changes involving the radiocarpal joint and by the shortening of the antebrachium that prevented the right tension and subsequent antigravity activity of the flexor carpi ulnaris muscle on the accessory carpal bone in the stance phase. Mild recurvatum of the radius could also play a role in carpal hyperextension. The degenerative changes of the radiocarpal joint may have been attributable to previous intramedullary pinning from the distal articular surface of the radius at the primary care clinic. Repair of radial diaphyseal fractures using intramedullary pins is not recommended because this technique has been associated with a high rate of postoperative complications. ${ }^{6}$

Other possible cause of lameness includes changes to radial head caused by previous surgeries and radioulnar synostosis at the site of fracture healing. Synostosis is a common complication of radioulnar fracture repair in dogs and cats. ${ }^{7}$ Even if the effects of this complication are unknown, in dogs the radius and ulna have an average of 15 degrees pronation and 17 degrees supination, respectively, during flexion and extension while walking and trotting on a treadmill and thus radioulnar synostosis abolishes physiological joint kinematics, possibly predisposing to lameness. ${ }^{50}$

In conclusion, internal fixation using a locking plate with proximal screws in the olecranon and distal screws in the radius can be considered an effective treatment method in dogs with proximal radial nonunion and a small proximal radial fragment.

\section{Conflict of Interest}

None declared.

\section{References}

1 Denny HR. Fracture of the radius and ulna. In: A Guide to Canine Orthopaedic Surgery 2nd ed. Boston: Blackwell Scientific Publications; 1985:179-185

2 Boudrieau RJ. Fractures of the radius and ulna. In: Slatter D, ed. Textbook of Small Animal Surgery. Vol 2. 3rd edition Philadelphia: WB Saunders; 2003:1953-1973

3 McCartney W, Kiss K, Robertson I. Treatment of distal radial/ulnar fractures in 17 toy breed dogs. Vet Rec 2010;166(14):430-432

4 Fox DB. Radius and ulna. In: Tobias KM, Johnston SA, eds. Veterinary Surgery: Small Animal. Vol 1 St Louis: Elsevier Saunders; 2012:760-784

5 Waters DJ, Breur GJ, Toombs JP. Treatment of common forelimb fractures in miniature and toy breed dogs. J Am Anim Hosp Assoc 1993;29:442

6 Lappin MR, Aron DN, Herron HL. Fractures of the radius and ulna in the dog. J Am Anim Hosp Assoc 1983;19(05):643-650

7 Piras L, Cappellari F, Peirone B, Ferretti A. Treatment of fractures of the distal radius and ulna in toy breed dogs with circular external skeletal fixation: a retrospective study. Vet Comp Orthop Traumatol 2011;24(03):228-235

8 Haas B, Reichler IM, Montavon PM. Use of the tubular external fixator in the treatment of distal radial and ulnar fractures in small dogs and cats. Vet Comp Orthop Traumatol 2003;3:132-137

9 Bierens D, Unis MD, Cabrera SY, Kass PH, Owen TJ, Mueller MG. Radius and ulna fracture repair with the IMEX miniature circular external skeletal fixation system in 37 small and toy breed dogs: a retrospective study. Vet Surg 2017;46(04):587-595

10 Hamilton MH, Langley Hobbs SJ. Use of the AO veterinary mini 'T'plate for stabilisation of distal radius and ulna fractures in toy breed dogs. Vet Comp Orthop Traumatol 2005;18(01):18-25

11 Gibert S, Ragetly GR, Boudrieau RJ. Locking compression plate stabilization of 20 distal radial and ulnar fractures in toy and miniature breed dogs. Vet Comp Orthop Traumatol 2015;28(06):441-447

12 De Arburn Parent R, Benamou J, Gatineau M, Clerfond P, Planté J Open reduction and cranial bone plate fixation of fractures involving the distal aspect of the radius and ulna in miniature- and toy- 
breed dogs: 102 cases (2008-2015). J Am Vet Med Assoc 2017;250 (12):1419-1426

13 Aikawa T, Miyazaki Y, Shimatsu T, lizuka K, Nishimura M. Clinical outcomes and complications after open reduction and internal fixation utilizing conventional plates in 65 distal radial and ulnar fractures of miniature- and toy-breed dogs. Vet Comp Orthop Traumatol 2018;31(03):214-217

14 Larsen LJ, Roush JK, McLaughlin RM. Bone plate fixation of distal radius and ulna fractures in small- and miniature-breed dogs. J Am Anim Hosp Assoc 1999;35(03):243-250

15 Saikku-Bäckström A, Räihä JE, Välimaa T, Tulamo RM. Repair of radial fractures in toy breed dogs with self-reinforced biodegradable bone plates, metal screws, and light-weight external coaptation. Vet Surg 2005;34(01):11-17

16 Muir P. Distal antebrachial fractures in toy-breed dogs. Compend Contin Educ Pract Vet 1997;19:137-145

17 Sumner-Smith G. A comparative investigation into the healing of fractures in miniature poodles and mongrel dogs. J Small Anim Pract 1974;15(05):323-328

18 Blaeser LL, Gallagher JG, Boudrieau RJ. Treatment of biologically inactive nonunions by a limited en bloc ostectomy and compression plate fixation: a review of 17 cases. Vet Surg 2003;32(01):91-100

19 Brinker MR. Nonunions: evaluation and treatment. In: Browner BD, Levine AM, Jupiter JB, et al, eds. Skeletal Trauma: Basic Science, Management, and Reconstruction. 3rd edition Philadelphia, PA: W.B. Saunders; 2003:507-604

20 Kaderly RE. Delayed union, nonunion and malunion. In: Slatter D, ed. Textbook of Small Animal Surgery. 2nd edition Philadelphia, PA: Saunders; 1993:1676-1685

21 Vertenten G, Gasthuys F, Cornelissen M, Schacht E, Vlaminck L. Enhancing bone healing and regeneration: present and future perspectives in veterinary orthopaedics. Vet Comp Orthop Traumatol 2010;23(03):153-162

22 Ragetly GR, Griffon DJ. The rationale behind novel bone grafting techniques in small animals. Vet Comp Orthop Traumatol 2011;24 (01):1-8

23 Faria MLE, Lu Y, Heaney K, Uthamanthil RK, Muir P, Markel MD. Recombinant human bone morphogenetic protein-2 in absorbable collagen sponge enhances bone healing of tibial osteotomies in dogs. Vet Surg 2007;36(02):122-131

24 Boudrieau RJ. Initial experience with rhBMP-2 delivered in a compressive resistant matrix for mandibular reconstruction in 5 dogs. Vet Surg 2015;44(04):443-458

25 Baltzer WI, Cooley S, Warnock JJ, Nemanic S, Stieger-Vanegas SM. Augmentation of diaphyseal fractures of the radius and ulna in toy breed dogs using a free autogenous omental graft and bone plating. Vet Comp Orthop Traumatol 2015;28(02):131-139

26 Hong HS. Ulnar radial nonunion fracture treated with recombinant human bone morphogenetic protein-2 in a dog. J Vet Clin Seoul 2001;18:156-159

27 Schmoekel H, Schense JC, Weber FE, et al. Bone healing in the rat and dog with nonglycosylated BMP-2 demonstrating low solubility in fibrin matrices. J Orthop Res 2004;22(02):376-381

28 Milovancev M, Muir P, Manley PA, Seeherman HJ, Schaefer S. Clinical application of recombinant human bone morphogenetic protein-2 in 4 dogs. Vet Surg 2007;36(02):132-140

29 Massie AM, Kapatkin AS, Fuller MC, Verstraete FJ, Arzi B. Outcome of nonunion fractures in dogs treated with fixation, compression resistant matrix, and recombinant human bone morphogenetic protein-2. Vet Comp Orthop Traumatol 2017;30(02):153-159

30 Bernard F, Furneaux R, Adrega Da Silva C, Bardet JF. Treatment with rhBMP-2 of extreme radial bone atrophy secondary to fracture management in an Italian Greyhound. Vet Comp Orthop Traumatol 2008;21(01):64-68
31 Gatineau M, Planté J Ulnar interlocking intramedullary nail stabilization of a proximal radio-ulnar fracture in a dog. Vet Surg 2010;39(08):1025-1029

32 Muir P, Manley PA. Stabilisation of fractures of the proximal radius and ulna in a dog by application of a single plate to the ulna. Vet Rec 1994;134(23):599-601

33 Piermattei DL, Johnson KA. An Atlas of Surgical Approaches to the Bones and Joints of the Dog and Cat. Fourth edition Philadelphia, PA: Elsevier; 2004:247-249

34 DeAngelis MP. Causes of delayed union and nonunion of fractures. Vet Clin North Am 1975;5(02):251-258

35 Brianza SZ, Delise M, Maddalena Ferraris M, D’Amelio P, Botti P. Cross-sectional geometrical properties of distal radius and ulna in large, medium and toy breed dogs. J Biomech 2006;39(02): 302-311

36 Johnston SA, Von Pfeil DJF, Dejardin LM, et al. Internal fracture fixation. In: Tobias KM, Johnston SA, eds. Veterinary Surgery: Small Animal. St. Louis, MO: Elsevier Saunders; 2018:654-690

37 Guerrero TG, Kalchofner K, Scherrer N, Kircher P. The Advanced Locking Plate System (ALPS): a retrospective evaluation in 71 small animal patients. Vet Surg 2014;43(02):127-135

38 Arens S, Schlegel U, Printzen G, Ziegler WJ, Perren SM, Hansis M. Influence of materials for fixation implants on local infection. An experimental study of steel versus titanium DCP in rabbits. J Bone Joint Surg Br 1996;78(04):647-651

39 Palmer MP, Altman DT, Altman GT, et al. Can we trust intraoperative culture results in nonunions? J Orthop Trauma 2014;28 (07):384-390

40 Barnhart MD, Rides CF, Kennedy SC, et al. Fracture repair using a polyaxial locking plate system (PAX). Vet Surg 2013;42(01): 60-66

41 Reddi AH, Huggins C. Biochemical sequences in the transformation of normal fibroblasts in adolescent rats. Proc Natl Acad Sci US A 1972;69(06):1601-1605

42 Reddi AH. Cell biology and biochemistry of endochondral bone development. Coll Relat Res 1981;1(02):209-226

43 Wozney JM, Rosen V, Celeste AJ, et al. Novel regulators of bone formation: molecular clones and activities. Science 1988;242 (4885):1528-1534

44 Itoh T, Mochizuki M, Nishimura R, et al. Repair of ulnar segmental defect by recombinant human bone morphogenetic protein-2 in dogs. J Vet Med Sci 1998;60(04):451-458

45 Suzuki Y, Montagne K, Nishihara A, Watabe T, Miyazono K. BMPs promote proliferation and migration of endothelial cells via stimulation of VEGF-A/VEGFR2 and angiopoietin-1/Tie2 signalling. J Biochem 2008;143(02):199-206

46 Moyen BJ, Lahey PJ Jr, Weinberg EH, Harris WH. Effects on intact femora of dogs of the application and removal of metal plates. A metabolic and structural study comparing stiffer and more flexible plates. J Bone Joint Surg Am 1978;60(07):940-947

47 Egger EL, Histand MB, Norrdin RW, et al. Canine Osteotomy Healing when stabilized with decreasingly rigid fixation compared to constantly rigid fixation. Vet Comp Orthop Traumatol 1993;6:182-187

48 Egger EL, Lewallen DG, Nordin RW, et al. Effects of destabilizing rigid external fixation on healing of unstable canine osteotomies. Trans Orthop Res Soc 1988;13:302

49 Franczuszki D, Chalman JA, Butler HC, et al. Postoperative effects of experimental femoral shortening in the mature dog. J Am Anim Hosp Assoc 1987;23:429-437

50 Guillou R, Dejardin L, McDonald C, et al.3-Dimensional Kinematics of the Normal Canine Elbow at The Walk and Trot, Proceedings, Annual Meeting of the Veterinary Orthopedic Society, Crested Butte, CO, March 3-10, 2012 